\title{
Reversible and Broad-Range Oxygen Sensing Based on Purely Organic Long-Lived Photoemitters
}

\author{
Efe Armagan, Kongchang Wei, Giuseppino Fortunato, Esther Amstad,* and René Michel Rossi*
}

Cite This: ACS Appl. Polym. Mater. 2021, 3, 2480-2488

Read Online

ABSTRACT: The detection of oxygen $\left(\mathrm{O}_{2}\right)$ by optical sensors is of growing importance, for example, in biology, life science, environmental science, and aerodynamics, where the composition of gases is crucial for many applications. Purely organic optical $\mathrm{O}_{2}$ sensors are very attractive for monitoring food deterioration, biosensing, and biomonitoring, as they can provide continuous, reversible, and nondestructive $\mathrm{O}_{2}$ sensing. However, purely organic optical reversible $\mathrm{O}_{2}$ sensors that can work with a broad $\mathrm{O}_{2}$ concentration range and that have robust reversibility are yet to be realized. We hereby developed a purely organic optical $\mathrm{O}_{2}$ sensor by embedding polyimide-based photoemitters

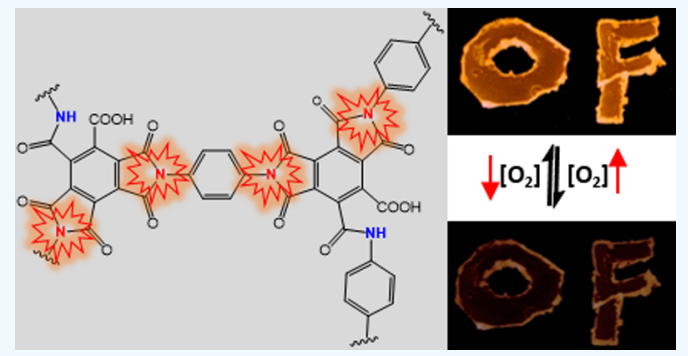
within a poly(vinyl alcohol) (PVA) matrix. The photoemitters are synthesized through one-pot hydrothermal reactions. They have a lifetime of room temperature phosphorescence (RTP) of up to a few microseconds $(\mu \mathrm{s})$ when they are embedded into the polymer matrix. Our results demonstrate that the photoemitters have higher sensitivity and broader sensing range to $\mathrm{O}_{2}$ if they are synthesized with longer reaction times because they possess a more rigid polyimide structure. The $\mathrm{O}_{2}$ sensor with such optimized photoemitters embedded in the polymer matrix exhibits continuous and broad-range $\mathrm{O}_{2}$ sensing in the range of $0-16 \% \mathrm{O}_{2}$. The Stern-Volmer quenching constant $\left(K_{\mathrm{sv}}\right)$ was calculated to be $0.2351 \mathrm{kPa}^{-1}$ for the linear response range of $0-4 \% \mathrm{O}_{2}$. Moreover, the $\mathrm{O}_{2}$ sensor can be repetitively used at least 10 times in the linear range $(0-$ $4 \% \mathrm{O}_{2}$ ) or beyond the linear range (up to $21 \% \mathrm{O}_{2}$ ). The metal-free, purely organic sensors that enable the continuous and repetitive detection of $\mathrm{O}_{2}$ within a relevant $\mathrm{O}_{2}$ sensing range are appealing especially for monitoring packaged food, biomonitoring, and biosensing.

KEYWORDS: room temperature phosphorescence, polyimide nanoparticles, organic optical oxygen sensor, broad-range oxygen sensing, reversible oxygen sensing

\section{INTRODUCTION}

Oxygen sensing has been a topic of intensive study in many fields, ${ }^{1}$ such as food monitoring, ${ }^{2}$ cell biology, ${ }^{3}$ biomedicine, ${ }^{4}$ and environmental monitoring. ${ }^{5}$ Particularly, optical $\mathrm{O}_{2}$ sensors have attracted much attention from many biological and medical applications, where the detection of $\mathrm{O}_{2}$ changes in a continuous, reversible, nontoxic, and nondestructive manner is in high demand. However, this is difficult to achieve with conventional optical $\mathrm{O}_{2}$ sensors due to their low photostability and high toxicity. ${ }^{6-9}$ The general principle behind the $\mathrm{O}_{2}$ response of optical $\mathrm{O}_{2}$ sensors is mostly the quenching of longlived emissions of photoemitters, such as phosphorescence and delayed fluorescence, due to the dynamic collision with $\mathrm{O}_{2}$ molecules. ${ }^{10}$ Long-lived emission states can be activated in the presence of efficient spin-orbit coupling (SOC), which enables intersystem crossing (ISC) between excited singlet states and excited triplet states. ${ }^{11}$ To promote the efficient SOC and therefore trigger an $\mathrm{O}_{2}$ response, most conventional optical $\mathrm{O}_{2}$ sensors have a photoemitter containing a metal ion, such as $\mathrm{Ru}(\mathrm{II}), \mathrm{Ln}(\mathrm{III}), \mathrm{Pt}(\mathrm{II})$, and $\mathrm{Pd}(\mathrm{II}) .{ }^{12-14}$ For example, Ln(III)based optical $\mathrm{O}_{2}$ sensors display excellent reversibility in a $0-$ $100 \% \mathrm{O}_{2}$ sensing range. ${ }^{15}$ However, these sensors are toxic due to an interchange between these metal ions and the cations in the biological systems $\left(\mathrm{Ca}^{2+}, \mathrm{Fe}^{2+}, \mathrm{Mg}^{2+}\right.$, and $\left.\mathrm{Na}^{+}\right) .{ }^{16-18}$ To address this shortcoming, metal-free optical $\mathrm{O}_{2}$ sensors based on organic long-lived photoemitters have been developed. ${ }^{19,20}$

Purely organic photoemitters with room temperature phosphorescence (RTP) are promising materials for $\mathrm{O}_{2}$ sensing, thanks to their low cost, ease of production, and efficient recombination pathway at room temperature. RTP has been observed when heteroatoms of purely organic photoemitters are bonded to aromatic compounds such as aromatic diketones, ${ }^{21}$ diacids, diesters, ${ }^{22}$ and diamines ${ }^{23}$ or functionalized with heavy halogen atoms such as chlorine, ${ }^{24}$ bromine, $^{25}$ and iodine. ${ }^{26}$ The quantum yield (QY) of such RTP photoemitters can be improved by inhibiting their internal molecular motions in various ways, including the

Received: January 15, 2021

Accepted: March 24, 2021

Published: April 5, 2021 
development of crystallization-enhanced systems, ${ }^{22,27}$ the induction of aggregation-induced intersystem crossings, ${ }^{28}$ the encapsulation of organic photoemitters within rigid cages, ${ }^{29}$ and structural rigidification through covalent ${ }^{30}$ or noncovalent interactions. ${ }^{31-33}$ Although a wide range of purely organic long-lived photoemitters with RTP have been developed, only few studies have investigated their $\mathrm{O}_{2}$ sensing performance. $^{34-36}$ For example, various phosphorescence QYs were achieved with a difluoroboron dibenzoylmethane-poly(lactic acid) solid-state sensor where the heavy halogen atom effect was employed, resulting in a linear dependency on the $\mathrm{O}_{2}$ concentration up to $1 \% \mathrm{O}_{2}$. However, the $\mathrm{O}_{2}$ calibration curve displays nonlinearity beyond $1 \% \mathrm{O}_{2}$ concentration, rendering the broad-range calibration within an $\mathrm{O}_{2}$ concentration of 1$100 \%$ difficult. $^{37}$ DeRosa et al. developed difluoroboron $\beta$ diketonate polylactide photoemitters that display a linear dependency on the $\mathrm{O}_{2}$ concentration within the full range if functionalized with iodide (I). ${ }^{38}$ However, $\beta$-diketonate-based compounds can undergo photodegradation during UV exposure, leading to the poor photostability of these luminophores and hence poor sensing reversibility. ${ }^{39}$

Recently, our group reported purely organic para-phenylenediamine (PPD)-based photoemitters embedded in a highmolecular-weight poly(vinyl alcohol) (PVA) matrix. Their fluorescence is quenched within the range of $0-8 \% \mathrm{O}_{2}{ }^{40}$ However, the nonlinear calibration curve and unsatisfactory reversibility have hampered their further use. In this study, we introduce a new purely organic solid-state optical $\mathrm{O}_{2}$ sensor composed of polyimide-based RTP photoemitters, which are embedded in a PVA matrix to repetitively enhance the $\mathrm{O}_{2}$ response. The photoemitters were synthesized from mellitic acid (MA) and PPD by using polyimide chemistry and were thus named MA-PPD. Thanks to the structural rigidity and strong intermolecular interactions, polyimides have an improved RTP quantum yield. ${ }^{41-43}$ In particular, we embedded the most efficient RTP photoemitter, MA-PPD6, in a PVA matrix that inhibits the intermolecular motion via the abundance of hydrogen bond donor and acceptor groups, thereby improving phosphorescence emission. The resulting $\mathrm{O}_{2}$ sensor can reversibly detect $\mathrm{O}_{2}$ in the range of $0-16 \%$ without significant losses in sensitivity: it has a linear dependence on the $\mathrm{O}_{2}$ concentration in the range of $0-4 \%$ $\mathrm{O}_{2}$ and a nonlinear dependency in the range of $4-16 \% \mathrm{O}_{2}$.

\section{EXPERIMENTAL}

Materials. PPD ( $\geq 99 \%)$ and mellitic acid (MA) ( $\geq 99 \%)$ were purchased from Sigma-Aldrich (St. Louis, MO, USA) and Tokyo Chemical Industry (Tokyo, Japan), respectively, for the synthesis of luminophores. Ultra-pure water (Milli-Q) was used as a solvent for the reactions. Potassium bromide $(\mathrm{KBr})$ was obtained from Merck (Darmstadt, Germany) for the pellet preparation. The PVA polymer (Mowiol 40-88, MW 205,000 Da) was obtained from SigmaAldrich. Oxygen $\left(\mathrm{O}_{2}\right)(99.99 \%)$, nitrogen $\left(\mathrm{N}_{2}\right)(99.8 \%)$, and carbon dioxide $\left(\mathrm{CO}_{2}\right)$ (99.99\%) were purchased from Carbagas (Zurich, Switzerland) for the sensor performance studies.

Methods. Synthesis of the Luminophores. A PPD and MA precursor mixture $(1 \mathrm{mg} / \mathrm{mL})$ was prepared in a 3:1 molar ratio in ultra-pure water. The prepared mixture solution was vigorously stirred for $30 \mathrm{~min}$ at room temperature. Subsequently, the solution was transferred to a polytetrafluoroethylene (PTFE)-lined synthesis autoclave reactor (Parr Instrument, Acid Digestion Vessel) with a volume of $50 \mathrm{~mL}$. The autoclave reactor was placed in a preheated oven (Series ED Avantgarde Line, BINDER, Spain) at $180{ }^{\circ} \mathrm{C}$ for different reaction times. The products were then dried in a freezedryer (Alpha 3-4 LSC basic, Martin Christ, Germany) for $24 \mathrm{~h}$, resulting in MA-PPD $x$, where MA, PPD, and $x$ represent mellitic acid, para-phenylenediamine, and reaction time (in hours), respectively.

Characterization Techniques. Fourier transform infrared (FT-IR) spectra were obtained with a Varian-640-IR (Portmann Instruments $\mathrm{GmbH}$, Biel-Benken, Switzerland) operated in the wavenumber range of $400-4000 \mathrm{~cm}^{-1}$ with a $2 \mathrm{~cm}^{-1}$ spectral resolution. The pellets were prepared by mixing $0.05 \% \mathrm{w} / \mathrm{w} \mathrm{KBr}$ into the MA-PPD $x$ powder for the quantitative FT-IR analysis.

The relative imide/amide content obtained from quantitative FTIR analysis was calculated as follows: the $\mathrm{C}-\mathrm{N}$ peak of the amide and imide group was deconvoluted in the wavenumber range of 1350$1450 \mathrm{~cm}^{-1}$ using Gaussian fitting with a multicurve peak analyzer in OriginLab. Afterward, the area under each deconvoluted peak was calculated with the integration tool in OriginLab to obtain the relative imide/amide content. ${ }^{44}$

X-ray photoelectron spectroscopy (XPS) was performed on a PHI VersaProbe II (Physical Electronics, Chanhassen, MN, USA) to investigate the chemical composition of the luminophores. The spectra were recorded with the energy resolution of $0.8 \mathrm{eV} /$ step and pass energy of $187.85 \mathrm{eV}$ for the survey scan, and the energy resolution of $0.125 \mathrm{eV} /$ step and pass energy of $29.35 \mathrm{eV}$ for highresolution scans. Carbon $1 \mathrm{~s}$ at $284.5 \mathrm{eV}$ was used as a reference to correct the charge effects. The samples were prepared by pressing MA-PPD $x$ powders onto an indium foil (Alfa Aesar, 99.99\% purity, Kandel, Germany).

The relative imide/amide content obtained from quantitative XPS analysis was calculated as follows: The data were analyzed using CasaXPS (Casa Software Ltd., UK). The N1s peaks were deconvoluted into three main peaks corresponding to amide, imide, and protonated $\mathrm{N}$ functionalities. ${ }^{45,46}$ The area under each deconvoluted peak was calculated to determine the relative imide/ amide abundance.

Prior to the high-resolution transmission electron microscopy (HRTEM) measurement, $0.05 \%(\mathrm{w} / \mathrm{v})$ of a MA-PPD $x$ solution was dropcasted on TEM grids (carbon film coated, 400 mesh, $\mathrm{Cu}$ ) (Electron Microscopy Sciences, PA, USA). The HR-TEM images were obtained with a JEOL JEM-1400 Plus (JEOL Ltd., Tokyo, Japan) operated at an acceleration voltage of $120 \mathrm{kV}$ with the detector of the JEOL CCD camera Ruby.

The photoluminescence (PL) of MA-PPD $x$ luminophores in an aqueous medium or a polymer matrix was measured with a Horiba Jobin Yvon FluoroMax-4 (Horiba Jobin Yvon GmbH, Bensheim, Germany). To determine the concentration for the highest PL intensity, a concentration series of $0.005,0.01,0.02,0.03,0.05,0.1$, 0.2 , and $0.5 \%(\mathrm{w} / \mathrm{v})$ was used for all MA-PPD $x$. The excitation range of $280-600 \mathrm{~nm}$ and emission range of $300-800 \mathrm{~nm}$ were used with the slit width of $3 \mathrm{~nm}$.

The lifetime of all MA-PPD $x$ was quantified with a Horiba DeltaPro (Horiba Jobin Yvon GmbH, Bensheim, Germany) at the excitation wavelength of $510 \mathrm{~nm}$. A LUDOX solution was used to calculate the instrument response factor (IRF).

Fabrication of the PVA/MA-PPDx Film. The solution was prepared by adding $0.05 \%(\mathrm{w} / \mathrm{v})$ of MA-PPD $x$ and $5 \%$ PVA powder (Mowiol 40-88) to ultra-pure water. Subsequently, the mixture was vigorously stirred at $60{ }^{\circ} \mathrm{C}$ for $12 \mathrm{~h}$. One milliliter of the solution was dropped on a polystyrene Petri dish and dried at $50{ }^{\circ} \mathrm{C}$ for $24 \mathrm{~h}$ to evaporate the water.

$\mathrm{O}_{2}$ Sensor Performance Test of the PVA/MA-PPDx Film. To test the oxygen sensitivity, a custom-built environmental chamber located in the spectrafluorometer chamber was purged with an $\mathrm{O}_{2} / \mathrm{N}_{2}$ gas mixture containing $0,1,2,4,8,12,16$, and $21 \% \mathrm{O}_{2}$. The gas fluxes were controlled with a flow-mixer setup (OxiQuant $\mathrm{S}$, Envitec $\mathrm{GmbH}$, Wismar, Germany). Specifically, before each measurement, the film was kept at $100 \% \mathrm{~N}_{2}$ flow for $20 \mathrm{~min}$. Subsequently, it was purged with the targeted $\mathrm{O}_{2} / \mathrm{N}_{2}$ mixture for another $20 \mathrm{~min}$. Photoluminescence measurements were obtained at the desired $\mathrm{O}_{2} / \mathrm{N}_{2}$ ratio by exciting the sensors in a range of $280-600 \mathrm{~nm}$ and recording an emission spectrum in the range of $300-800 \mathrm{~nm}$.

The lifetime analysis was performed with a Horiba DeltaPro (Horiba Jobin Yvon GmbH, Bensheim, Germany) for all PVA/MA- 
A<smiles>Nc1ccc(N)cc1</smiles>

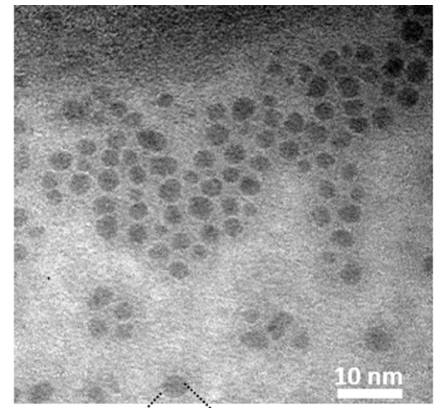

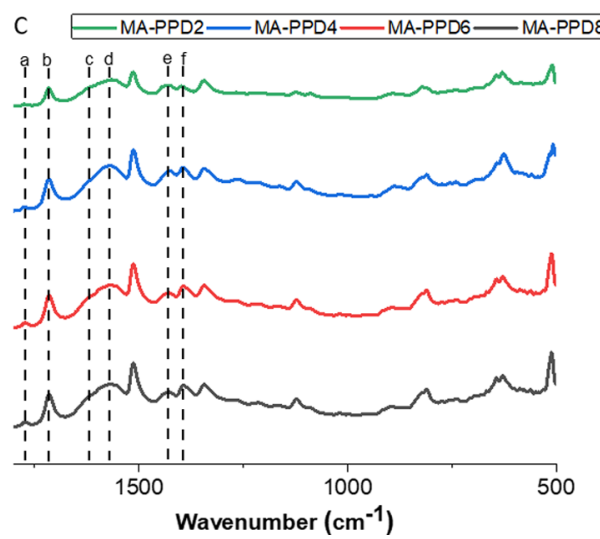

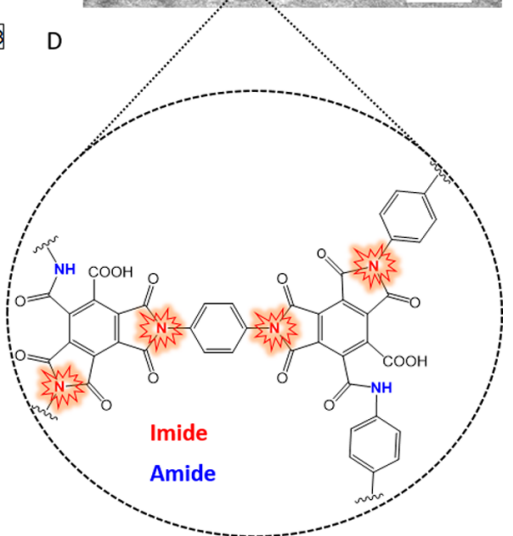

Figure 1. Synthesis and characterization of MA-PPD luminophores. (a) Reaction scheme between PPD and mellitic acid, (b) TEM image of MAPDD6, (c) FT-IR spectra of MA-PPDx, and (d) the chemical structure of the MA-PPD luminophores.

Table 1. Imide/Amide Relative Content and PL Lifetime of MA-PPDx Luminophores

\begin{tabular}{|c|c|c|c|c|c|}
\hline \multirow[b]{2}{*}{ sample name } & \multirow[b]{2}{*}{ reaction time $(\mathrm{h})$} & \multicolumn{2}{|c|}{ imide/amide relative content } & \multicolumn{2}{|c|}{ lifetime $(\mu \mathrm{s})^{a}$} \\
\hline & & FT-IR & XPS & {$\left[\mathrm{O}_{2}\right]=0 \%$} & {$\left[\mathrm{O}_{2}\right]=21 \%$} \\
\hline MA-PPD2 & 2 & $0.73 \pm 0.04$ & $1.19 \pm 0.16$ & $4.51 \pm 0.22$ & $2.83 \pm 0.08$ \\
\hline MA-PPD4 & 4 & $1.17 \pm 0.08$ & $2.69 \pm 0.23$ & $5.81 \pm 0.46$ & $3.22 \pm 0.41$ \\
\hline MA-PPD6 & 6 & $1.39 \pm 0.09$ & $10.13 \pm 0.18$ & $17.07 \pm 0.72$ & $5.61 \pm 0.29$ \\
\hline MA-PPD8 & 8 & $1.38 \pm 0.05$ & $10.02 \pm 0.29$ & $16.38 \pm 0.55$ & $5.58 \pm 0.45$ \\
\hline
\end{tabular}

${ }^{a}$ For lifetime measurements, MA-PPD $x$ luminophores were embedded in the PVA matrix.

PPD $x$ films at the excitation wavelength of $510 \mathrm{~nm}$ under a standard air and anoxic environment $\left(100 \% \mathrm{~N}_{2}\right)$.

To test the sensor reversibility, the $\mathrm{O}_{2}$ concentration in the custombuilt chamber was switched between 0 and $4 \% \mathrm{O}_{2}$ or between 0 and $21 \% \mathrm{O}_{2}$ for 10 times. The measurement chamber was purged with a mixture of $\mathrm{N}_{2} / \mathrm{CO}_{2}$ at the ratio of $60: 40$ or $\mathrm{N}_{2} / \mathrm{CO}_{2}$ at the ratio of 100:0 to investigate the sensor cross-sensitivity toward $\mathrm{CO}_{2}$. Similarly, the chamber was respectively kept under 80 and $20 \%$ relative humidity level at room temperature while purging with a constant $\mathrm{N}_{2}$ / $\mathrm{O}_{2}$ flow at the ratio of 79:21 to test the cross-interference of humidity.

\section{RESULTS AND DISCUSSION}

Chemical Characterization of the MA-PPD Photoemitters. MA-PPD photoemitters were synthesized for different durations as described in the "Methods" section and according to the literature ${ }^{45}$ (Figure 1a and Table 1). The photoemitters synthesized with different reaction times, named MA-PPD $x$ with $x$ indicating the reaction time in hours, were evaluated based on chemical and optical characterization. Nanometer-sized particles with diameters of $3 \mathrm{~nm}$ were synthesized at a constant precursor concentration of $1 \mathrm{mg} /$ $\mathrm{mL},{ }^{44}$ as shown in the HR-TEM images in Figure $1 \mathrm{~b}$ and Figure S1 and summarized in Table S1. Note that the reaction time has no effect on the average size of the photoemitters, indicating that the nanoparticles reach the final size in the first $2 \mathrm{~h}$.

To understand the evolution of the functional groups and investigate the time-dependent polyimide formation, we performed FT-IR on our photoemitters. All the peaks observed in MA-PPD2 were preserved as the reaction proceeded up to 8 $\mathrm{h}$, as shown in Figure 1c and summarized in Table S2. This result indicates that all types of the functional groups are formed within the first $2 \mathrm{~h}$. The peak at $1775 \mathrm{~cm}^{-1}$ (a) and $1718 \mathrm{~cm}^{-1}(\mathrm{~b})$, attributed to the symmetric and asymmetric carbonyl $(\mathrm{C}=\mathrm{O})$ stretching, showed the successful imide formation. The presence of the peaks corresponding to the $\mathrm{C}=\mathrm{O}$ stretching of the amide bond and $\mathrm{N}-\mathrm{H}$ in-plane bending of amide at $1627 \mathrm{~cm}^{-1}$ (c) and $1556 \mathrm{~cm}^{-1}$ (d) indicates that the final structure is composed of a mixture of imide and amide bonds (Figure 1d). To quantify the ratio of imide and amide bonds, we analyze the intensities of the $\mathrm{C}-\mathrm{N}$ stretching vibrations located at $1422 \mathrm{~cm}^{-1}$ (e) and $1385 \mathrm{~cm}^{-1}$ (f). The intensities of the $\mathrm{C}-\mathrm{N}$ stretching vibration peak characteristic for imides increased, whereas those of the $\mathrm{C}-\mathrm{N}$ stretching vibration peak of amides decreased, until the reaction time reached $6 \mathrm{~h}$. Indeed, the relative imide/amide content increased by $90.4 \%$ when the reaction time was prolonged from 2 to $6 \mathrm{~h}$ and plateaued thereafter as shown in 

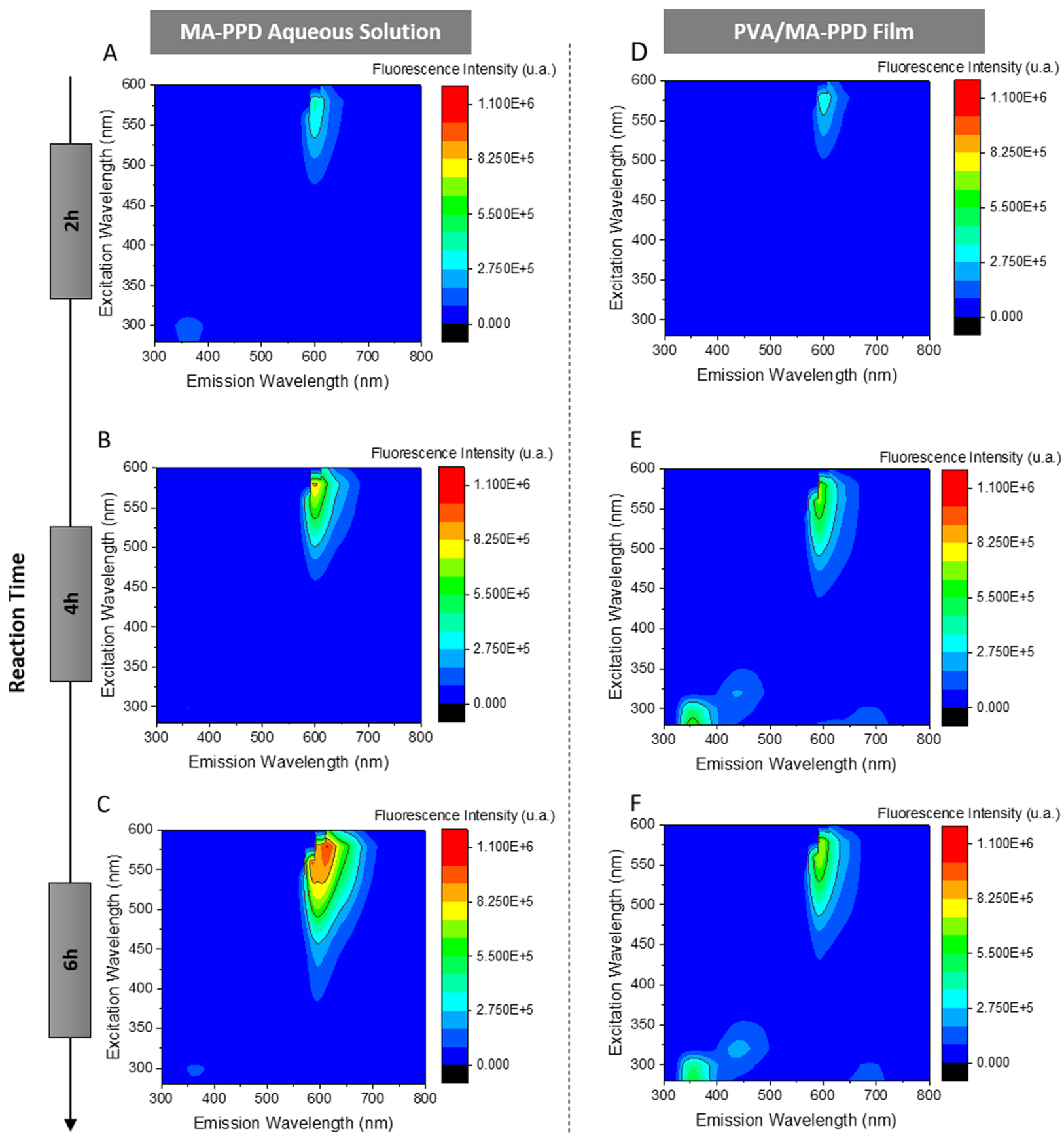

Figure 2. Photoluminescence contour plots of MA-PPD $x$ as a function of their synthesis time $(x=2,4$, or $6 \mathrm{~h})$. (a-c) MA-PPD $x$ in aqueous solutions. (d-f) MA-PPDx embedded in PVA films.

Table 1. This result indicates that the imide formation within MA-PPD nanoparticles is terminated within $6 \mathrm{~h}$.

To investigate the evolution of the elemental composition of the functional groups contained in the MA-PPD $x$ samples, we quantified the concentration of each chemical element with XPS. The elemental analysis shows no significant change in the carbon concentration. By contrast, the nitrogen concentration increases from 5.5 to $11.1 \%$, whereas the oxygen concentration decreases from 31.6 to $25.6 \%$, as the reaction proceeds from 2 to $6 \mathrm{~h}$ (Table S3). No significant change was observed at longer reaction times $(8 \mathrm{~h})$, well in agreement with our FT-IR analysis. The decrease in the elemental oxygen concentration might be due to the loss of the hydroxyl $(-\mathrm{OH})$ group during the imide formation (Figure 1d). To combine the elemental composition analysis with the deconvoluted high-resolution XPS, we decomposed the C1s peak into four peaks at 284.4, $285.4,287.3$, and $288.2 \mathrm{eV}$ that we attributed to the $\mathrm{sp}^{2}$ carbon, $\mathrm{C}-\mathrm{N}$ group, carbon (amide), and carbon (imide), respectively. ${ }^{47}$ To quantify the evolution of imide groups, the $\mathrm{N} 1 s$ peaks were deconvoluted into three main peaks corresponding to amide $\left(-\mathrm{CONH}_{2} /-\mathrm{CONHR} /-\mathrm{CONR}\right)$ $(398.9 \mathrm{eV})$, imide $(399.8 \mathrm{eV})$, and protonated $\mathrm{N}(401.3 \mathrm{eV})$ functionalities. ${ }^{45,46}$ This analysis reveals that the relative imide/amide ratio increases from 1.19 to 10.13 as the reaction proceeds from 2 to $6 \mathrm{~h}$, plateauing thereafter (Table 1). These results are in excellent agreement with our FTIR results.

Optical Characterization of the MA-PPD Photoemitters. To investigate the effect of imide formation on 

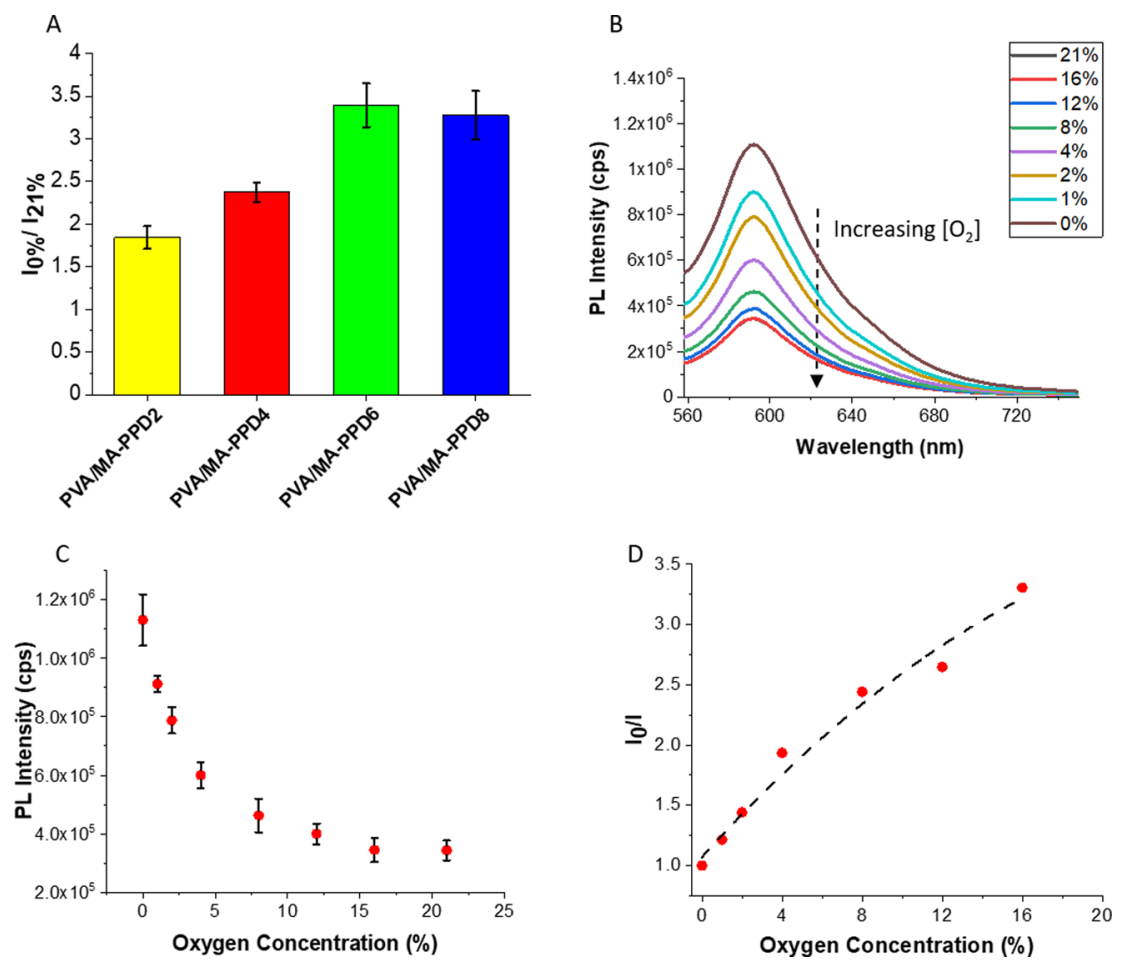

Figure 3. Oxygen sensing performance of PVA/MA-PPD sensors. (a) Relative PL intensity of PVA/MA-PPD $x$ sensors between 0 and $21 \% \mathrm{O}_{2}$, (b) PL spectra of the PVA/MA-PPD6 sensor at different $\mathrm{O}_{2}$ concentrations, (c) maximum PL intensity of the PVA/MA-PPD6 sensor at different $\mathrm{O}_{2}$ concentrations, and (d) Stern-Volmer plot of the PVA/MA-PPD6 sensor.

photoluminescence behavior, we recorded the photoluminescence spectra of MA-PPDx samples dispersed in aqueous solutions. We limit our characterization to samples synthesized during 2-6 $\mathrm{h}$ because we did not observe any significant difference in their composition thereafter anymore. We did not observe any significant positional change in the photoluminescence spectra $\left(\lambda_{\mathrm{ex}}=585 \mathrm{~nm}, \lambda_{\mathrm{em}}=609 \mathrm{~nm}\right)$ when samples were excited between 280 and $600 \mathrm{~nm}$, as shown in Figure $2 \mathrm{a}-\mathrm{c}$. If excited at the wavelength of $\lambda_{\mathrm{ex}}=585 \mathrm{~nm}$, the spectra of aqueous solutions displayed a red emissive behavior, which is consistent with other polyimide luminophores. ${ }^{42,43}$ The charge-transfer (CT) interaction between electrondonating amines and electron-accepting carboxyl has been reported to lower the energy gap between the excited and ground state, resulting in red photoemission. It is noteworthy that, while the position of the emission peak is preserved, the photoluminescence (PL) intensity increased from $332,311 \mathrm{cps}$ to $1,002,938 \mathrm{cps}$ as the reaction proceeds from 2 to $6 \mathrm{~h}$. This result suggests that the increase in PL intensity of MA-PPD is related to the new imide groups that form at longer reaction times.

To understand the effect of the microenvironment on the optical properties of MA-PPD, we recorded the photoluminescence spectra of MA-PPD nanoparticles embedded within a high-molecular-weight PVA matrix (Figure 2d-f). Compared to MA-PPD in aqueous solutions $\left(\lambda_{\mathrm{em}}=609 \mathrm{~nm}\right)$, slightly blue-shifted emissive states $\left(\lambda_{\mathrm{em}}=597 \mathrm{~nm}\right)$ were observed when excited at $570 \mathrm{~nm}$. The emissive behavior $\left(\lambda_{\mathrm{em}}\right.$ $=597 \mathrm{~nm}$ ) of MA-PPD embedded within the PVA matrix is in good agreement with the reported spectral characteristics of polyimide-based luminescent systems. ${ }^{42,43}$ The blue-shift has been observed in a similar system where luminophores synthesized by phenylenediamine were encapsulated within a
PVA matrix. This behavior was ascribed to the absence of the solvent-relaxation effect. ${ }^{48}$ Similar to MA-PPD dispersed in aqueous solutions, we did not identify any significant positional shift of the emissive peaks from MA-PPD $x(x=2,4$, or 6$)$ nanoparticles if embedded in PVA films (Figure $2 \mathrm{~d}-\mathrm{f}$ ). This result demonstrates that the interaction between the polyimide groups of MA-PPD photoemitters with water and the PVA matrix has negligible effects on the intrinsic optical properties and chemical structure of MA-PPD nanoparticles.

$\mathrm{O}_{2}$ Sensing Performance. The key characteristic of $\mathrm{O}_{2}$ sensors is their $\mathrm{O}_{2}$-dependent photoluminescence. To quantify this parameter, we dispersed MA-PPD in an aqueous solution or embedded them in a PVA film and recorded their photoluminescence spectra at oxygen concentrations of 0 and $21 \%$. Aqueous solutions of MA-PPD nanoparticles did not show any $\mathrm{O}_{2}$-concentration-dependent photoluminescence, as shown in Figure S2. By contrast, MA-PPD nanoparticles embedded in a PVA film displayed a strongly increased PL intensity with decreasing oxygen concentration. To quantify this increase, we determined the ratio between the PL intensity at 0 and $21 \% \mathrm{O}_{2}$ concentration as $I_{0 \%} / I_{21 \%}$. For PVA/MAPPD2, PVA/MA-PPD4, PVA/MA-PPD6, and PVA/MAPPD8 samples, $I_{0 \%} / I_{21 \%}$ was $1.84,2.37,3.39$, and 3.27 , respectively (Figure 3a). The maximum $I_{0 \%} / I_{21 \%}$ value of 3.39 is significantly higher than that of the previously reported organic optical $\mathrm{O}_{2}$ sensor with the sensing range of $0-21 \%$ $\mathrm{O}_{2} \cdot{ }^{34,49,50}$ Such a clear $\mathrm{O}_{2}$-dependent photoluminescence might be due to the strong intermolecular interactions between embedded nanoparticles and the polymer matrix, which barely affect the MA-PPD chemical structure but eliminate the nonradiative triplet-singlet ground-state recombination. These results demonstrate the advantage of embedding MA-PPD nanoparticles in a PVA matrix if they are used as $\mathrm{O}_{2}$ sensors. 

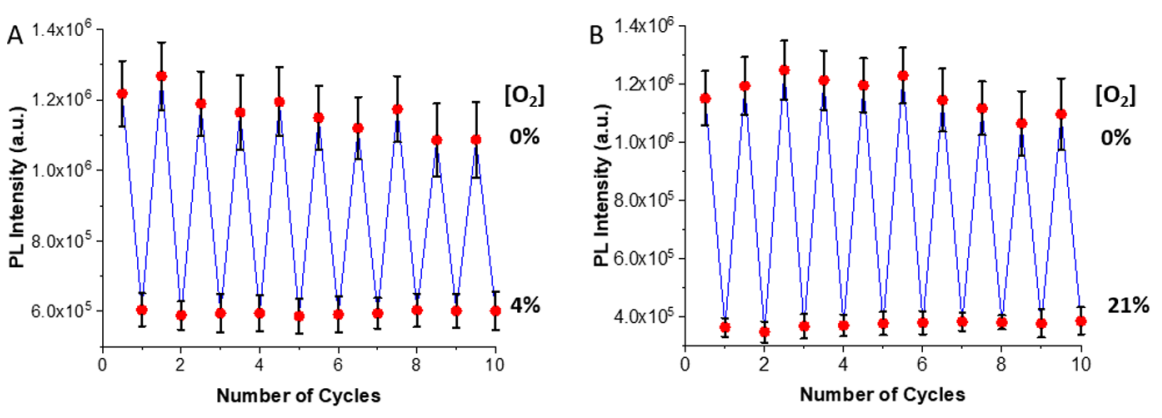

C

D
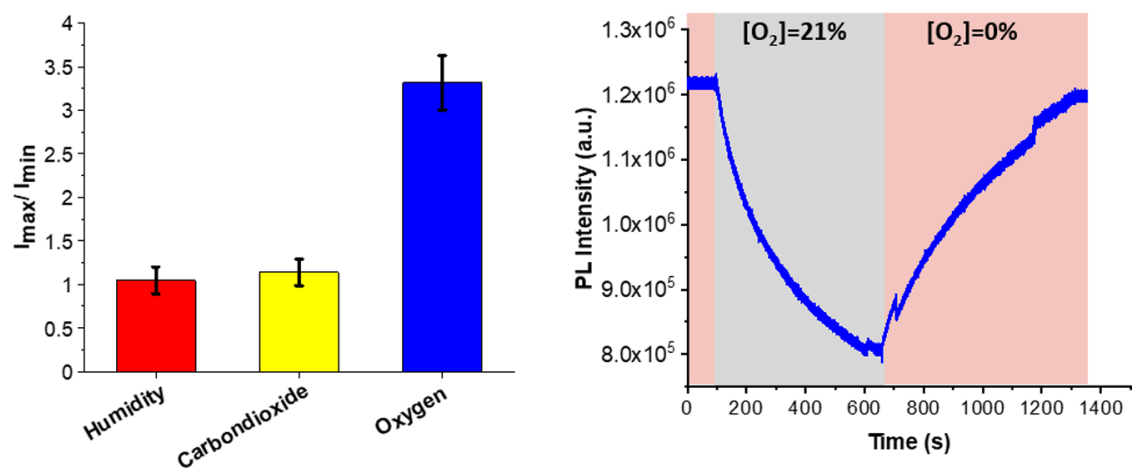

Figure 4. $\mathrm{O}_{2}$ sensing features of the PVA/MA-PPD6 sensor. (a-b) Sensing reversibility of the PVA/MA-PPD6 sensor exposed to 10 concentration cycles of $0-4 \%$ or $0-21 \% \mathrm{O}_{2}$, (C) sensing cross-interference of humidity and $\mathrm{CO}_{2}$ toward $\mathrm{O}_{2}$, and (d) time-dependent sensing response to a $0-$ $21-0 \% \mathrm{O}_{2}$ cycle.

Our results indicate that MA-PPD-containing PVA matrices are excellent $\mathrm{O}_{2}$ sensors. To understand the $\mathrm{O}_{2}$ sensing mechanism, we quantify the PL lifetime as a function of the $\mathrm{O}_{2}$ concentration within a range of $0-21 \% \mathrm{O}_{2}$, as summarized in Table 1. At $21 \% \mathrm{O}_{2}$, all samples exhibit room temperature phosphorescence (RTP) with a lifetime value $(\tau)$ of a few microseconds (Figure S3). The short phosphorescence lifetime indicates the presence of an efficient RTP mechanism, as previously shown. ${ }^{39}$ Based on these results, we quantified the PL lifetime ratio $\tau_{0 \%} / \tau_{21 \%}$, where $\tau_{0 \%}$ and $\tau_{21 \%}$ indicate the PL lifetime measured at 0 and $21 \% \mathrm{O}_{2}$, respectively. This ratio was $1.59,1.81,3.04$, and 2.93 for PVA/MA-PPD2, PVA/MAPPD4, PVA/MA-PPD6, and PVA/MA-PPD8 sensors, respectively. Note that the lifetime ratios of the last two samples are very similar, in good agreement with our FTIR results, confirming that the composition of MA-PPD remains unchanged when the synthesis time exceeds $6 \mathrm{~h}$. Combining this result with those of the FT-IR and XPS analysis, we note that, within the first $6 \mathrm{~h}$ of reaction time, the $\mathrm{O}_{2}$ response of MA-PPD increases with its formation time and plateaus thereafter. This increased efficiency can be attributed to the increased conformational rigidity of MA-PPD nanoparticles that eliminates nonradiative recombinations, thereby enabling efficient $\mathrm{O}_{2}$ RTP quenching.

To quantify the $\mathrm{O}_{2}$ sensing performance, we analyzed the chemically most stable and performing sample, namely, PVA/ MA-PPD6. We measured the emission spectra within an $\mathrm{O}_{2}$ concentration range of $0-21 \%$, as shown in Figure $3 \mathrm{~b}$. The emissive state of MA-PPD6 was quenched until the $\mathrm{O}_{2}$ concentration reached 16\%, as shown in Figure 3c, indicating that our sensors can be used within this $\mathrm{O}_{2}$ range. Note that the $\mathrm{O}_{2}$ sensing range has previously been much narrower: similar systems could only detect $\mathrm{O}_{2}$ in the range of $0-8 \%{ }^{40}$ We assign the broader $\mathrm{O}_{2}$ sensing range $(0-16 \%)$ of our sensor to the efficient RTP mechanism that shortens the phosphorescence lifetime, thereby enabling $\mathrm{O}_{2}$ quenching at higher $\mathrm{O}_{2}$ concentrations. ${ }^{38}$ To quantify the sensitivity, we fitted the Stern-Volmer plot to a first-order polynomial function between 0 and $4 \% \mathrm{O}_{2}\left(R^{2}=0.998\right.$, Figure S4). The limit of detection (LOD) was calculated according to the extrapolated concentration at which the signal is three times the averaged standard deviation $(3 \sigma)$ of the fluorescence intensity. A detection limit of $950 \mathrm{ppm}$ was achieved with good linearity in the range of $0-4 \% \mathrm{O}_{2}$. The good quality of the fits indicates that our sensors display a linear response to the $\mathrm{O}_{2}$ concentration up to $4 \%$. It has been previously discussed that the linear calibration curve is desirable for simple one-point instrument calibrations that enable the use of the standard calibration cards for the sensors. ${ }^{51}$ The Stern-Volmer quenching constant was calculated in accordance with the Stern-Volmer equation.

$$
I_{0} / I=1+K_{\mathrm{sv}} \cdot\left[\mathrm{O}_{2}\right]
$$

where $I_{0}$ and $I$ represent the luminescence intensities in the absence and presence of $\mathrm{O}_{2}$, respectively. $K_{\mathrm{sv}}$ is the SternVolmer quenching constant, and $\left[\mathrm{O}_{2}\right]$ is the oxygen concentration in the environment. The Stern-Volmer constant $\left(K_{\mathrm{sv}}\right)$ was calculated to be $0.2351 \mathrm{kPa}^{-1}$ for the linear response range of $0-4 \% \mathrm{O}_{2}$. It is also noteworthy that, within a broader range of $\mathrm{O}_{2}$ concentration $(0-21 \%)$, instead of linear fitting, the second-order polynomial function fits the data well $\left(R^{2}=0.986\right.$, Figure $3 \mathrm{~d}$ and Figure S4), indicating a nonlinear response above $4 \% \mathrm{O}_{2}$. This behavior could be 
attributed to the inhomogeneity of the microenvironment in the polymer film. ${ }^{52}$

The usefulness of $\mathrm{O}_{2}$ sensors strongly depends on their ability to repetitively sense $\mathrm{O}_{2}$ without significant losses in sensitivity. To assess the reversibility of our $\mathrm{O}_{2}$ sensors, we cycled the $\mathrm{O}_{2}$ concentration between 0 and $4 \%$ for 10 times, as shown in Figure $4 a$, and similarly between 0 and $21 \%$ also for 10 times, as shown in Figure 4b. We did not observe any significant decay in the sensitivity, indicating that no noticeable photo-oxidation processes occur within 10 cycles (Figure S8).

The reliability of the $\mathrm{O}_{2}$ sensors is crucial for developing marketable sensors. To assess the reproducibility of our $\mathrm{O}_{2}$ sensors, we measured the sensor-to-sensor reproducibility with five different sensors fabricated under the same experimental conditions. The variation of $I_{0 \%} / I_{21 \%}$ of the fabricated sensors was less than $2 \%$ (Figure S10a). Indeed, this variation of PL intensity is statistically insignificant. Batch-to-batch reproducibility was assessed by characterizing five batches containing five different sensors for each batch. The maximum variation of $I_{0 \%} / I_{21 \%}$ between different batches was $9.8 \%$ (Figure S10b). These results indicate that the PVA/MA-PPD6 sensor possesses the best sensor-to-sensor reproducibility, as shown in Figure S10a. This good reproducibility enables the operation of our sensors in the batch calibration mode.

$\mathrm{O}_{2}$ sensors are only truly useful if they also operate in the presence of other types of gases. To test the influence of other atmospheric parameters such as humidity and $\mathrm{CO}_{2}$, we quantified the sensing performance of our sensors in the presence of 20 and $80 \%$ relative humidity or in the presence of 0 and $60 \% \mathrm{CO}_{2}$, as shown in Figure 4c. We did not observe any significant PL response of the MA-PPD6 sensor to humidity or $\mathrm{CO}_{2}$, as revealed by the ratio between maximum and minimum PL intensity $\left(I_{\max } / I_{\min } \sim 1\right)$ when changing the humidity or $\mathrm{CO}_{2}$ within the range of $20-80 \%$ relative humidity and $0-60 \% \mathrm{CO}_{2}$, respectively (Figure S9). This result indicates that cross-interferences between $\mathrm{O}_{2}$ and humidity or $\mathrm{CO}_{2}$ can be neglected in our PVA/MA-PPD6 sensors.

The temperature dependence of our $\mathrm{O}_{2}$ sensor shows a slight positive slope as the temperature increases (Figure S6a). For the linear response range $\left(0-4 \% \mathrm{O}_{2}\right)$, the temperaturedependent Stern-Volmer constant change $\left(\Delta K_{\mathrm{sv}} / \Delta T\right)$ was found to be $0.0077 \mathrm{kPa}^{-1}{ }^{\circ} \mathrm{C}^{-1}$ for the temperature range of $5-30{ }^{\circ} \mathrm{C}$ (Figure S6b). The constant value enables the simple correction for the sensor calibration at different temperatures. The positive temperature coefficient of PVA/MA-PPD6 sensor is in good agreement with the previously fabricated optical $\mathrm{O}_{2}$ sensors. ${ }^{53,54}$ The increase in $\mathrm{O}_{2}$ sensitivity with respect to the temperature could be attributed to several reasons including (1) the solubility of oxygen in the PVA matrix, (2) the diffusion rate of oxygen into the PVA matrix, and (3) the dynamic quenching constant. ${ }^{55}$

To test the speed of the sensor to $\mathrm{O}_{2}$ concentration change, the response time of the PVA/MA-PPD6 sensors was measured within one cycle of $0-21-0 \% \mathrm{O}_{2}$. Upon exposure to $20 \% \mathrm{O}_{2}$, the time required to reduce the PL intensity by 95\% $\left(\Delta t_{95 \min }\right)$ was $427 \mathrm{~s}$, as shown in Figure $4 \mathrm{~d}$. The long response time is due to the low oxygen permeability of the PVA matrix and significant film thickness $(\sim 100 \mu \mathrm{m})$ that hinders $\mathrm{O}_{2}$ molecules from reaching the photoemitters. ${ }^{56}$ Although the long response time limits the application area of our sensors, we believe that the continuous, broad-range, and reversible sensing makes our purely organic $\mathrm{O}_{2}$ sensors promising for the detection of $\mathrm{O}_{2}$, for example, in packaged food where fast response times are not critical.

\section{CONCLUSIONS}

A purely organic rigid optical $\mathrm{O}_{2}$ sensor was developed by embedding polyimide-based nanoparticles within a highmolecular-weight PVA matrix. Our results indicate that the sensitivity of the composite strongly depends on the concentration of the imides present in the photoemitters and increases with increasing reaction time. If the photoemitters are embedded in a PVA matrix, they strongly interact with it, thereby limiting the nonradiative recombination, such that their photoluminescence is efficiently RTP quenched by $\mathrm{O}_{2}$ molecules. Our PVA/MA-PPD6 sensors can monitor $\mathrm{O}_{2}$ in a broad range up to concentrations of $16 \%$ with a linear response between 0 and $4 \% \mathrm{O}_{2}$ and nonlinear response between 4 and $16 \% \mathrm{O}_{2}$. Moreover, such sensors are resistant to photobleaching and atmospheric inference, making them promising materials for the detection of $\mathrm{O}_{2}$ in the field of food packaging.

\section{ASSOCIATED CONTENT}

\section{Supporting Information}

The Supporting Information is available free of charge at https://pubs.acs.org/doi/10.1021/acsapm.1c00064.

High-resolution transmission electron microscopy images (Figure S1); fluorescence contour plot of MAPPD6 nanoparticles in an aqueous solution (Figure S2); lifetime decay curve of MA-PPD6 nanoparticles embedded in a PVA film (Figure S3); Stern-Volmer fitting of RTP quenching (Figure S4, Figure S4-Note); representative PL spectra for the reversibility test (figure S5); temperature dependence of the $\mathrm{O}_{2}$ sensitivity of MA-PPD6/PVA (Figure S6); photobleaching of different probes at continuous illumination between 0 and $500 \mathrm{~s}$ (Figure S7); $\mathrm{O}_{2}$ sensing reversibility of the PVA/ MA-PPD6 sensor (Figure S8); the effect of humidity on the sensitivity of the PVA/MA-PPD6 sensor (Figure S9); sensor reproducibility of the PVA/MA-PPD6 sensor (Figure S10); average nanoparticle size obtained by HR-TEM (Table S1); type of the bonds for each MA-PPD $x$ sample obtained by FT-IR (Table S2); type of the elements for each MA-PPD $x$ sample obtained by XPS (Table S3); imide/amide relative ratio obtained by FT-IR (Table S4); imide/amide relative ratio obtained by XPS (Table S5); maximum PL intensity (cps) of three different MA-PPD6/PVA films (Table S6); second-order polynomial fitting details in the nonlinear quenching region $\left(4-16 \% \mathrm{O}_{2}\right)$ (Table S7); first-order polynomial fitting details in the nonlinear quenching region $\left(0-4 \% \mathrm{O}_{2}\right)$ (Table S8) (PDF)

\section{AUTHOR INFORMATION}

\section{Corresponding Authors}

Esther Amstad - Institute of Materials, Ecole Polytechnique

Fédérale de Lausanne (EPFL), CH-1015 Lausanne,

Switzerland; 이이.org/0000-0002-9491-1010;

Email: esther.amstad@epfl.ch

René Michel Rossi - Empa, Swiss Federal Laboratories for Materials Science and Technology, Laboratory for Biomimetic Membranes and Textiles, 9014 St. Gallen, Switzerland; Department of Health Sciences and Technology, Eidgenössische Technische Hochschule Zürich (ETH Zurich), 
CH-8092 Zurich, Switzerland; 이이이.org/0000-0003-

0946-682X; Email: rene.rossi@empa.ch

\section{Authors}

Efe Armagan - Empa, Swiss Federal Laboratories for Materials Science and Technology, Laboratory for Biomimetic Membranes and Textiles, 9014 St. Gallen, Switzerland; Institute of Materials, Ecole Polytechnique Fédérale de Lausanne (EPFL), CH-1015 Lausanne, Switzerland

Kongchang Wei - Empa, Swiss Federal Laboratories for Materials Science and Technology, Laboratory for Biomimetic Membranes and Textiles, 9014 St. Gallen, Switzerland; (1) orcid.org/0000-0002-6555-2768

Giuseppino Fortunato - Empa, Swiss Federal Laboratories for Materials Science and Technology, Laboratory for Biomimetic Membranes and Textiles, 9014 St. Gallen, Switzerland; (1) orcid.org/0000-0002-3889-7816

Complete contact information is available at: https://pubs.acs.org/10.1021/acsapm.1c00064

\section{Author Contributions}

E.A., K.W., R.M.R., and E.Am. designed the research. E.A. performed the experiments including the nanoparticle synthesis, chemical characterization, optical characterization, and sensor performance test. E.A. and K.W. analyzed the data. G.F. performed the XPS measurement and data analysis. E.A. drafted the paper. K.W., R.M.R., and E.Am. edited the manuscript.

\section{Notes}

The authors declare no competing financial interest.

"This author passed away in June 2020.

\section{ACKNOWLEDGMENTS}

This research was funded by the Swiss Competence Center for Materials Science and Technology (CCMX). The authors thank Dr. Luciano F. Boesel and Dr. Claudio Toncelli for their valuable suggestions and Dr. Peng Zeng for important assistance in the HR-TEM analysis. The authors dedicate this paper to the memory of Dr. Giuseppino Fortunato.

\section{REFERENCES}

(1) Ramamoorthy, R.; Dutta, P. K.; Akbar, S. A. Oxygen Sensors : Materials , Methods, Designs and Applications. J. Mater. Sci. 2003, 38, 4271-4282.

(2) Yousefi, H.; Su, H. M.; Imani, S. M.; Alkhaldi, K.; Filipe, C. D.; Didar, T. F. Intelligent Food Packaging: A Review of Smart Sensing Technologies for Monitoring Food Quality. ACS Sensors 2019, 4, 808-821.

(3) Kaelin, W., Jr.; Ratcliffe, S. P.; Krebs, H. An Award to Oxygen Sensing. Nat. Biomed. Eng. 2019, 3, 843-844.

(4) Roussakis, E.; Li, Z.; Nichols, A. J.; Evans, C. L. Oxygen-Sensing Methods in Biomedicine from the Macroscale to the Microscale. Angew. Chem., Int. Ed. 2015, 54, 8340-8362.

(5) Meixner, H.; Gerblinger, J.; Fleischer, M. Sensors for Monitoring Environmental Pollution. Sens. Actuators, B 1993, 15, 45-54.

(6) Jones, P. F. On the Use of Phosphorescence Quenching for Determining Permeabilities of Polymeric Films to Gases. Polym. Lett. 1968, 6, 487-491.

(7) Bizzarri, A.; Koehler, H.; Cajlakovic, M.; Pasic, A.; Schaupp, L.; Klimant, I.; Ribitsch, V. Continuous Oxygen Monitoring in Subcutaneous Adipose Tissue Using Microdialysis. Anal. Chim. Acta 2006, 573-574, 48-56.

(8) Choi, M. F.; Hawkins, P. A Novel Oxygen and/or Carbon Dioxide-Sensitive Optical Transducer. Talanta 1995, 42, 483-492.
(9) Choi, M. F.; Hawkins, P. A Fibre-Optic Oxygen Sensor Based on Contact Charge-Transfer Absorption. Sens. Actuators, B 1996, 30, 167-171.

(10) Patterson, L. K.; Porter, G.; Topp, M. R. Oxygen Quenching of Singlet and Triplet States. Chem. Phys. Lett. 1970, 7, 612-614.

(11) Lawetz, V.; Orlandi, G.; Siebrand, W. Theory of Intersystem Crossing in Aromatic Hydrocarbons. J. Chem. Phys. 1972, 56, 40584072.

(12) Payne, S. J.; Fiore, G. L.; Fraser, C. L.; Demas, J. N. Luminescence Oxygen Sensor Based on a Ruthenium(II) Star Polymer Complex. Anal. Chem. 2010, 82, 917-921.

(13) Toncelli, C.; Arzhakova, O. V.; Dolgova, A.; Volynskii, A. L.; Bakeev, N. F.; Kerry, J. P.; Papkovsky, D. B. Oxygen-Sensitive Phosphorescent Nanomaterials Produced from High-Density Polyethylene Films by Local Solvent-Crazing. Anal. Chem. 2014, 86, 1917-1923.

(14) Finikova, O. S.; Cheprakov, A. V.; Vinogradov, S. A. Synthesis and Luminescence of Soluble Meso-Unsubstituted Tetrabenzo- and Tetranaphtho[2,3] Porphyrins. J. Org. Chem. 2005, 70, 9562-9572.

(15) Nakai, H.; Kuyama, M.; Seo, J.; Goto, T.; Matsumoto, T.; Ogo, S. Luminescent $\mathrm{Tb}(\mathrm{III})$ and $\mathrm{Sm}(\mathrm{III})$ Complexes with a 1,4,7Triazacyclononane-Based Tris-Aryloxide Ligand for High-Performance Oxygen Sensors. Dalt. Trans. 2017, 46, 9126-9130.

(16) Jaishankar, M.; Tseten, T.; Anbalagan, N.; Mathew, B. B.; Beeregowda, K. N. Toxicity, Mechanism and Health Effects of Some Heavy Metals. Interdiscip. Toxicol. 2014, 7, 60-72.

(17) Ripa, R. S.; Haack-Sørensen, M.; Kastrup, J.; Ekblond, A. Labeling, Human Mesenchymal Stromal Cells with Indium-111, SPECT Imaging. In Kretsinger, R. H.; Uversky, V. N.; Permyakov, E. A. (eds); Encyclopedia of Metalloproteins. Springer, New York, 2013, pp. 1061-1223, DOI: 10.1007/978-1-4614-1533-6 429.

(18) Wang, J.; Zhao, Z.; Zhou, S.; Zhang, X.; Bo, H. The Antitumor Effect and Toxicity of a Ruthenium(II) Complex in Vivo. Inorg. Chem. Commun. 2018, 87, 49-52.

(19) Zhi, J.; Zhou, Q.; Shi, H.; An, Z.; Huang, W. Organic Room Temperature Phosphorescence Materials for Biomedical Applications. Chem. - Asian J. 2020, 15, 947-957.

(20) Gan, N.; Shi, H.; An, Z.; Wei, H. Recent Advances in PolymerBased Metal-Free Room-Temperature Phosphorescent Materials. Adv. Funct. Mater. 2018, 28, 1802657.

(21) Yuan, W. Z.; Shen, X. Y.; Zhao, H.; Lam, J. W.; Tang, L.; Lu, P.; Wang, C.; Liu, Y.; Wang, Z.; Zheng, Q.; Sun, J. Z. CrystallizationInduced Phosphorescence of Pure Organic Luminogens at Room Temperature. J. Phys. Chem. C 2010, 114, 6090-6099.

(22) Gong, Y.; Zhao, L.; Peng, Q.; Fan, D.; Yuan, W. Z.; Zhang, Y.; Tang, B. Z. Crystallization-Induced Dual Emission from Metal- and Heavy Atom-Free Aromatic Acids and Esters. Chem. Sci. 2015, 6, $4438-4444$.

(23) Jiang, K.; Zhang, L.; Lu, J.; Xu, C.; Cai, C.; Lin, H. Triple-Mode Emission of Carbon Dots: Applications for Advanced Anti-Counterfeiting. Angew. Chem. 2016, 128, 7347-7351.

(24) Xiang, Y.; Zhao, Y.; Xu, N.; Gong, S.; Ni, F.; Wu, K.; Luo, J.; Xie, G.; Lu, Z. H.; Yang, C. Halogen-Induced Internal Heavy-Atom Effect Shortening the Emissive Lifetime and Improving the Fluorescence Efficiency of Thermally Activated Delayed Fluorescence Emitters. J. Mater. Chem. C 2017, 5, 12204-12210.

(25) Kwon, M. S.; Lee, D.; Seo, S.; Jung, J.; Kim, J. Tailoring Intermolecular Interactions for Efficient Room-Temperature Phosphorescence from Purely Organic Materials in Amorphous Polymer Matrices. Angew. Chem., Int. Ed. 2014, 53, 11177-11181.

(26) Wang, J.; Gu, X.; Ma, H.; Peng, Q.; Huang, X.; Zheng, X.; Sung, S. H.; Shan, G.; Lam, J. W.; Shuai, Z.; Tang, B. Z. A Facile Strategy for Realizing Room Temperature Phosphorescence and Single Molecule White Light Emission. Nat. Commun. 2018, 9, 1-9.

(27) Shimizu, M.; Shigitani, R.; Nakatani, M.; Kuwabara, K.; Miyake, Y.; Tajima, K.; Sakai, H.; Hasobe, T. Siloxy Group-Induced Highly Efficient Room Temperature Phosphorescence with Long Lifetime. J. Phys. Chem. C 2016, 120, 11631-11639. 
(28) Yang, L.; Wang, X.; Zhang, G.; Chen, X.; Zhang, G.; Jiang, J. Aggregation-Induced Intersystem Crossing: A Novel Strategy for Efficient Molecular Phosphorescence. Nanoscale 2016, 8, 1742217426.

(29) Chen, H.; Ma, X.; Wu, S.; Tian, H. A Rapidly Self-Healing Supramolecular Polymer Hydrogel with Photostimulated RoomTemperature Phosphorescence Responsiveness. Angew. Chem., Int. Ed. 2014, 53, 14149-14152.

(30) Kwon, M. S.; Yu, Y.; Coburn, C.; Phillips, A. W.; Chung, K.; Shanker, A.; Jung, J.; Kim, G.; Pipe, K.; Forrest, S. R.; Youk, J. H.; Gierschner, J.; Kim, J. Suppressing molecular motions for enhanced room-temperature phosphorescence of metal-free organic materials. Nat. Commun. 2015, 6, 8947-8955.

(31) Tan, J.; Zhang, J.; Li, W.; Zhang, L.; Yue, D. Synthesis of Amphiphilic Carbon Quantum Dots with Phosphorescence Properties and Their Multifunctional Applications. J. Mater. Chem. C 2016, 4, 10146-10153.

(32) Tan, J.; Zou, R.; Zhang, J.; Li, W.; Zhang, L.; Yue, D. LargeScale Synthesis of N-Doped Carbon Quantum Dots and Their Phosphorescence Properties in a Polyurethane Matrix. Nanoscale 2016, 8, 4742-4747.

(33) Deng, Y.; Zhao, D.; Chen, X.; Wang, F.; Song, H.; Shen, D. Long Lifetime Pure Organic Phosphorescence Based on Water Soluble Carbon Dots. Chem. Commun. 2013, 49, 5751-5753.

(34) Charlesworth, J. M. Optical Sensing of Oxygen Using Phosphorescence Quenching. Sens. Actuators, B 1994, 22, 1-5.

(35) Chan, M. A.; Lawless, J. L.; Lam, S. K.; Lo, D. Fiber Optic Oxygen Sensor Based on Phosphorescence Quenching of Erythrosin B Trapped in Silica-Gel Glasses. Anal. Chim. Acta 2000, 408, 33-37.

(36) Badía, R.; Díaz-García, M. E.; García-Fresnadillo, A. A Sensitive Probe for Oxygen Sensing in Gas Mixtures, Based on RoomTemperature Phosphorescence Quenching. Microchim. Acta 1995, $121,51-61$.

(37) Zhang, G.; Palmer, G. M.; Dewhirst, M. W.; Fraser, C. L. A Dual-Emissive-Materials Design Concept Enables Tumour Hypoxia Imaging. Nat. Mater. 2009, 8, 747-751.

(38) DeRosa, C. A.; Seaman, S. A.; Mathew, A. S.; Gorick, C. M.; Fan, Z.; Demas, J. N.; Peirce, S. M.; Fraser, C. L. Oxygen Sensing Difluoroboron $\beta$-Diketonate Polylactide Materials with Tunable Dynamic Ranges for Wound Imaging. ACS Sensors 2016, 1, 13661373.

(39) Sayre, R. M.; Dowdy, J. C.; Gerwig, A. J.; Shields, W. J.; Lloyd, R. V. Unexpected Photolysis of the Sunscreen Octinoxate in the Presence of the Sunscreen Avobenzone. Photochem. Photobiol. 2005, $81,452-456$

(40) Armagan, E.; Thiyagarajan, S.; Wei, K.; Gursoy, A.; Fortunato, G.; Amstad, E.; Rossi, R. M.; Toncelli, C. Reversible Oxygen Sensing Based on Multi-Emission Fluorescence Quenching. Sensors 2020, 20, 477-490.

(41) Ronova, I. A.; Bruma, M.; Schmidt, H. W. Conformational Rigidity and Dielectric Properties of Polyimides. Struct. Chem. 2012, 23, 219-226.

(42) Kanosue, K.; Ando, S. Polyimides with Heavy Halogens Exhibiting Room-Temperature Phosphorescence with Very Large Stokes Shifts. ACS Macro Lett. 2016, 5, 1301-1305.

(43) Kanosue, K.; Hirata, S.; Vacha, M.; Augulis, R.; Gulbinas, V.; Ishige, R.; Ando, S. A Colorless Semi-Aromatic Polyimide Derived from a Sterically Hindered Bromine-Substituted Dianhydride Exhibiting Dual Fluorescence and Phosphorescence Emission. Mater. Chem. Front. 2019, 3, 39-49.

(44) Kim, T.; Park, B.; Lee, K. M.; Joo, S. H.; Kang, M. S.; Yoo, W. C.; Kwak, S. K.; Kim, B. S. Hydrothermal Synthesis of Compositionand Morphology-Tunable Polyimide-Based Microparticles. ACS Macro Lett. 2018, 7, 1480-1485.

(45) Zeng, D. W.; Yung, K. C.; Xie, C. S. XPS Investigation of the Chemical Characteristics of Kapton Films Ablated by a Pulsed TEA CO2 Laser. Surf. Coatings Technol. 2002, 153, 210-216.

(46) Yang, G.; Han, H.; Du, C.; Luo, Z.; Wang, Y. Facile Synthesis of Melamine-Based Porous Polymer Networks and Their Application for Removal of Aqueous Mercury Ions. Polymer 2010, 51, 61936202.

(47) Barshilia, H. C.; Ananth, A.; Gupta, N.; Anandan, C. Superhydrophobic Nanostructured Kapton ${ }^{\circledR}$ Surfaces Fabricated through Ar + O 2 Plasma Treatment: Effects of Different Environments on Wetting Behaviour. Appl. Surf. Sci. 2013, 268, 464-471.

(48) Jiang, K.; Zhang, L.; Lu, J.; Xu, C.; Cai, C.; Lin, H. Triple-Mode Emission of Carbon Dots: Applications for Advanced Anti-Counterfeiting. Angew. Chem., Int. Ed. 2016, 55, 7231-7235.

(49) Peterson, J. I.; Fitzgerald, R. V. Fiber-Optic Probe for in Vivo Measurement of Oxygen Partial Pressure. Anal. Chem. 1984, 56, 6267.

(50) Sharma, A.; Wolfbeis, O. S. Unusually Efficient Quenching of the Fluorescence of an Energy Transfer-Based Optical Sensor for Oxygen. Anal. Chim. Acta 1988, 212, 261-265.

(51) Kelly, C. A.; Toncelli, C.; Cruz-Romero, M.; Arzhakova, O. V.; Kerry, J. P.; Papkovsky, D. B. Phosphorescent O2 sensors integrated in polymeric film materials bylocal solvent crazing. Mater. Des. 2015, 77, 110-113.

(52) Zhang, G.; Chen, J.; Payne, S. J.; Kooi, S. E.; Demas, J. N.; Fraser, C. L. Multi-Emissive Difluoroboron Dibenzoylmethane Polylactide Exhibiting Intense Fluorescence and Oxygen-Sensitive Room-Temperature Phosphorescence. J. Am. Chem. Soc. 2007, 129, 8942-8943.

(53) Chu, C. S.; Lin, C. A. Optical fiber sensor for dual sensing of temperature and oxygen basedon PtTFPP/CF embedded in sol-gel matrix. Sens. Actuators, B 2014, 195, 259-265.

(54) Bittig, H. C.; Körtzinger, A.; Neill, C.; van Ooijen, E.; Plant, J. N.; Hahn, J.; Johnson, K. S.; Yang, B.; Emerson, S. R. Oxygen Optode Sensors: Principle, Characterization, Calibration, and Application in the Ocean. Front. Mar. Sci. 2018, 4, 429.

(55) Lakowicz, J. R.; Weber, G. Quenching of Fluorescence by Oxygen. A probe for Structural Fluctuations in Macromolecules. Biochemistry 1973, 12, 4161-4170.

(56) Gaume, J.; Wong-Wah-Chung, P.; Rivaton, A.; Thérias, S.; Gardette, J. L. Photochemical Behavior of PVA as an Oxygen-Barrier Polymer for Solar Cell Encapsulation. RSC Adv. 2011, 1, 1471-1481. 